\title{
Evaluation of locally manufactured patient-specific custom made implants for cranial defects using a silicone mould
}

\author{
AJ Vlok, S Naidoo, AS Kamat, D Lamprecht \\ Stellenbosch University \\ Corresponding author: Adriaan JVlok(ianvlok@sun.ac.za)
}

\begin{abstract}
Background: Cranial vault defects can pose a significant problem for neurosurgeons where autologous bone is no longer available for cranioplasty. Numerous materials exist to create implants which include polymethyl methacrylate (PMMA) and titanium. A technique using 3-dimensional CT scan reconstruction of a cranial defect and creating a silicon mould which can be autoclaved in theatre to create a PMMA implant was developed.

Objectives: The aim of this study is to evaluate the efficacy, cosmetic result, safety and cost-effectiveness of this procedure and compare this to existing techniques.

Methods: An ambispective study was performed in patients requiring cranioplasty with a custom made implant. Patients were assessed for risk factors and cosmetic outcome, surgical technique was described and complications and cost compared to existing literature between 2010 and 2016.

Results: Thirty retrospective and 30 consecutive prospective patients were recruited into the study. Overall sepsis rate was $8.3 \%$. All septic cases had superficial sepsis of which 2 grafts were removed due to cerebrospinal fluid leakage resulting in wound breakdown. A $100 \%$ accurate implant to defect ratio was achieved leading to a high satisfaction rate. Average cost was 5 times cheaper than the closest market related product.

Conclusion: Patient specific moulds using PMMA to create custom implants are safe, have excellent cosmetic results and are a very cost-effective option to manage cranial defects. Accurate planning strategies for large craniotomies, where bone will potentially be discarded, add to surgical effectiveness and cost-saving to the patient.
\end{abstract}

\section{Introduction}

Neurosurgeons are commonly faced with cranial vault defects. These often result from trauma in the form of skull fractures leading to bone loss or decompressive craniectomies to control raised intracranial pressure. Tumour infiltration into bone leads to excised bone being discarded as is the case in sepsis and osteitis which requires extensive debridement.

Several strategies exist to manage these defects. Saving the patient's own bone and replacing it at a later stage as an autologous cranioplasty is the preferred method. This can be achieved by storing the patient's bone in an appropriate bone freezer, or even storing it in a subcutaneous pouch created in the patient's abdomen. ${ }^{1,2}$

When replacing the patient's own bone is impossible, alternate strategies to close the cranial defects include covering the defect with a wide variety of materials such as titanium mesh, polymethyl methacrylate (PMMA) or hydroxyapatite. ${ }^{3}$ The challenges posed by these materials involve the expense of the material, sepsis risk, thermal injury to the brain and cosmetic defects when the original skull contours are not achieved.
PMMA or bone cement, as it is known in the orthopaedic realm, is readily available and cost effective and thus an attractive option to fill cranial vault defects. PMMA is an acrylic polymer created when two sterile components (a powder and a liquid) are mixed. A paste is formed and over the course of a few minutes will solidify. An exothermic reaction takes place during the curing of the polymer and can reach up to $86^{\circ} \mathrm{C} .{ }^{4}$ While the polymer sets, it can be moulded into a specific shape. ${ }^{5}$ Once hardened and cooled, it is safe for implant into humans. ${ }^{5,6}$ The exothermic curing process of the PMMA poses a challenge for use in cranioplasty as the paste cannot be held against the cranial defect to mould due to risk of thermal injury to the brain. ${ }^{2}$ The extreme temperatures also create difficulty in handling the paste, often leading to unsatisfactory cosmetic results.

The solution to this involves creating patient specific implants by using a computed tomogram (CT) 3-dimensional (3D) reconstruction of the defect. ${ }^{3,4}$ These implants are manufactured specific to the defect and from a myriad of materials of which titanium and PMMA are the most popular. 
In the South African market, limited access to these exist and therefore most implants are imported which leads to greater expense and increased turn-around time. Other international institutions identified these challenges and circumvented them by using the craniotomy bone intraoperatively to create a mirror implant with PMMA at the time of initial surgery, store it and autoclave when ready for implant. ${ }^{5,6}$ This approach, however, becomes impractical in a high trauma environment where it is difficult to predict who will eventually require cranioplasty.

A locally manufactured patient-specific custom-made silicone mould was pioneered at the Department of Neurosurgery at Tygerberg Academic Hospital in 2008. It involves creating a silicon mould as a negative of the desired implant based on a 3D reconstructed image of the defect, which can be autoclaved. This provides the surgeon with the opportunity to create the custom made implant in theatre under sterile conditions during the cranioplasty procedure.

A further advantage is to create a 3D printed model of the skull preoperatively, plan the surgery and craniotomy defect (often in tumour surgery) and create a template to draw out the proposed craniotomy during live surgery, as well as create the implant mould of the planned defect. This allows for immediate reconstruction during surgery, should it have been anticipated that the bone be discarded (see Figures 4,5,6).

The aim of this study is to perform a pro- and retrospective review of 60 locally manufactured patient specific moulds. The primary objective will be to assess the practicality, safety and cost effectiveness of the moulds and to compare the results to existing products and techniques. Specific outcome measures include cost, cosmesis and sepsis rates compared to the existing body of knowledge.

Ethical approval for this study was obtained from the Health Research Ethics Committee at the University of Stellenbosch under the number S13/08/143.

\section{Materials and methods}

A total of 30 consecutive patients were included in the prospective leg of the study that required custom made cranial implants at Tygerberg Academic Hospital in the Western Cape Province of South Africa.

The data collected included primary pathology, patient demographics, duration between pathology and cranioplasty, surgical and postoperative complications, efficacy and accuracy of the implant, intraoperative detail and patient satisfaction (dissatisfied, neutral or satisfied).

The retrospective review studied 30 patients for implant accuracy and complications.

Septic complications were classified as superficial or deep.

The custom-made silicon moulds were made by Craniotech $^{\circledR}$, a locally based company specialised in patient specific cranial reconstruction and 3D printing solutions (See Figures 1,2 and 4).

The silicon mould is autoclaved just prior to the procedure and presented to the surgeon once the defect is exposed. Dural edges are freed until the full thickness of the skull surrounding

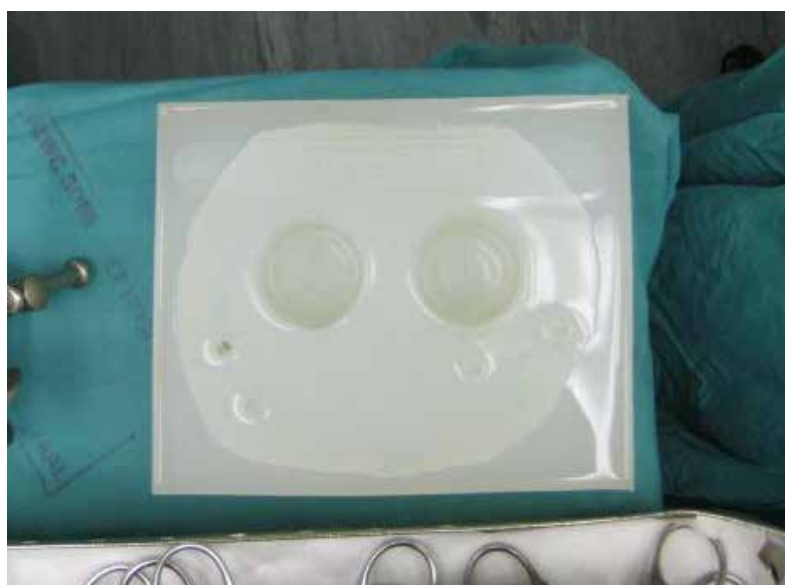

Figure 1: A two piece custom silicon mould that can be autoclaved.

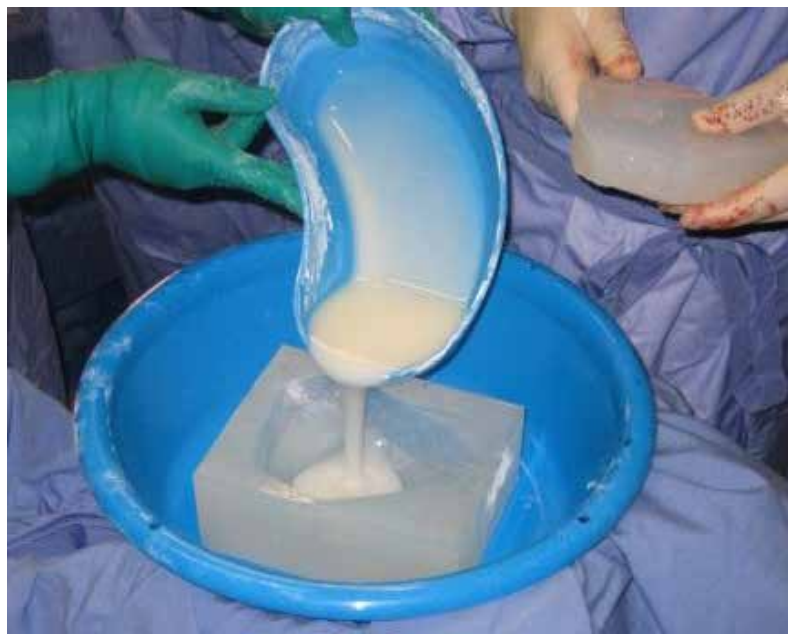

Figure 2: PMMA is mixed and placed in the mould.

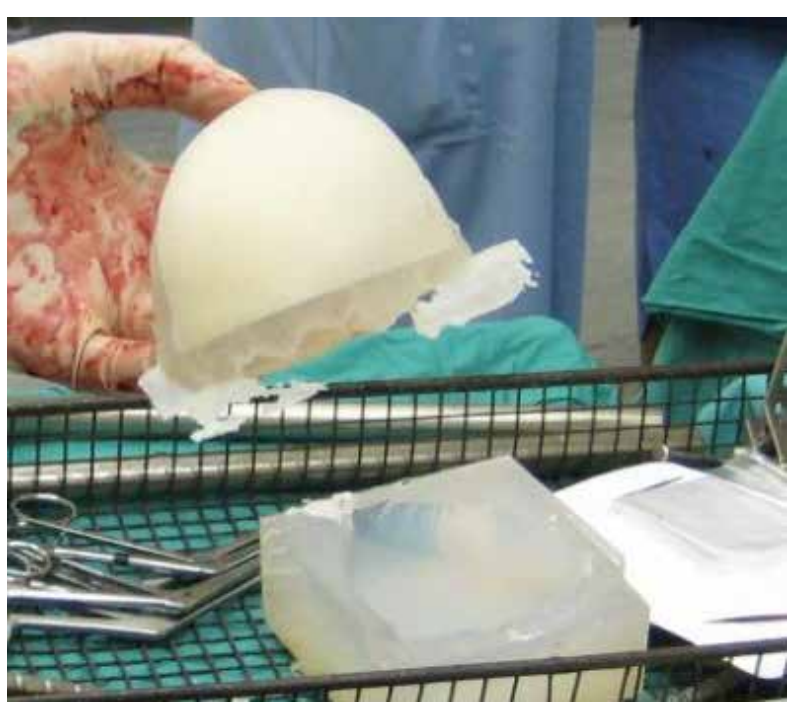

Figure 3: A custom implant. Excess PMMA is easily trimmed.

the defect is appreciated. PMMA is mixed and placed in the mould. After it has set and cooled, minor trimming of the graft is done and secured in place with cranial fixators and/or miniplates. Prophylactic antibiotics are given routinely prior 


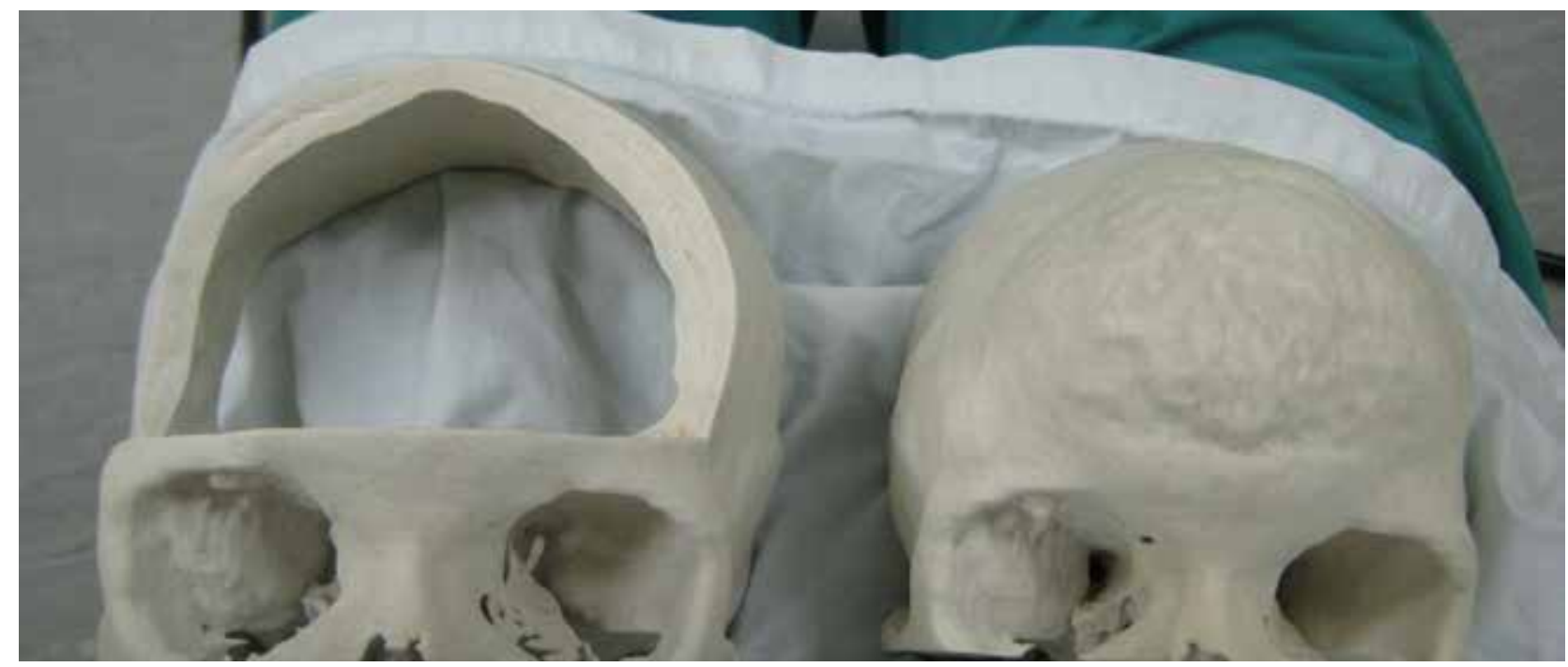

Figure 4: Pre-surgical planning of an intraosseous meningioma. A planned craniotomy is done on the 3D model.

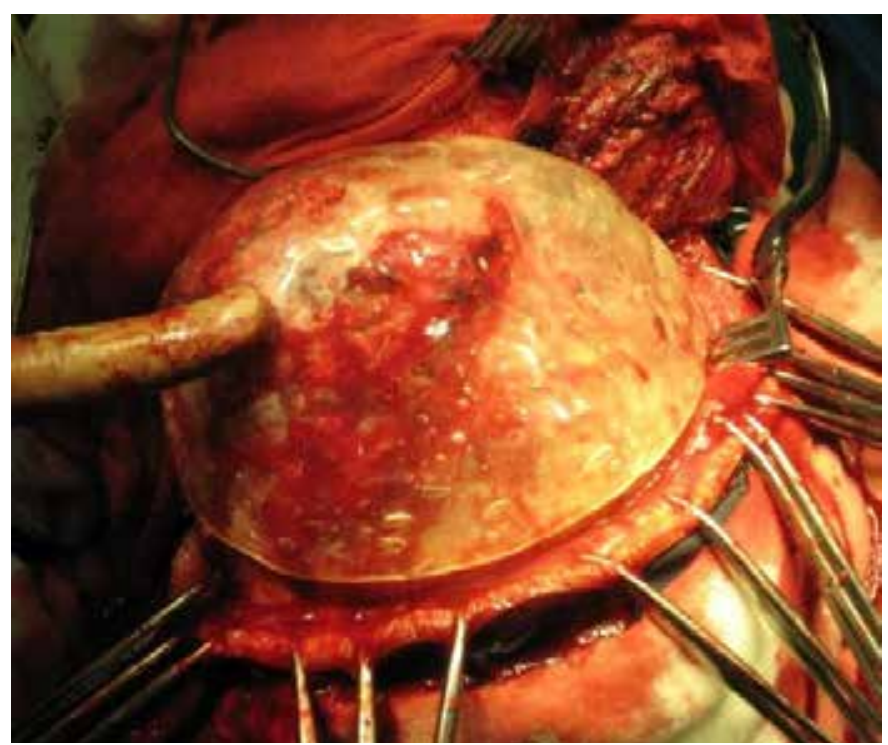

Figure 5: A planning template of the craniotomy is made and used intraoperatively to guide the actual craniotomy.

to the first incision. The defect is routinely closed in available layers.

The primary outcome was described by means of proportion of patients with a specific complication (with 95\% Confidence Intervals indicating population estimates).

Numeric data has been described using means (with Standard Deviations) or medians (with Interquartile range) if the data is skewed.

\section{Results}

Between 2010 and 2016, 60 patients underwent PMMA cranioplasty using a custom-made silicon mould. All patients had their implants produced intraoperatively using the templated moulding system. Thirty of these patients were analysed retrospectively and 30 consecutive cases prospectively.

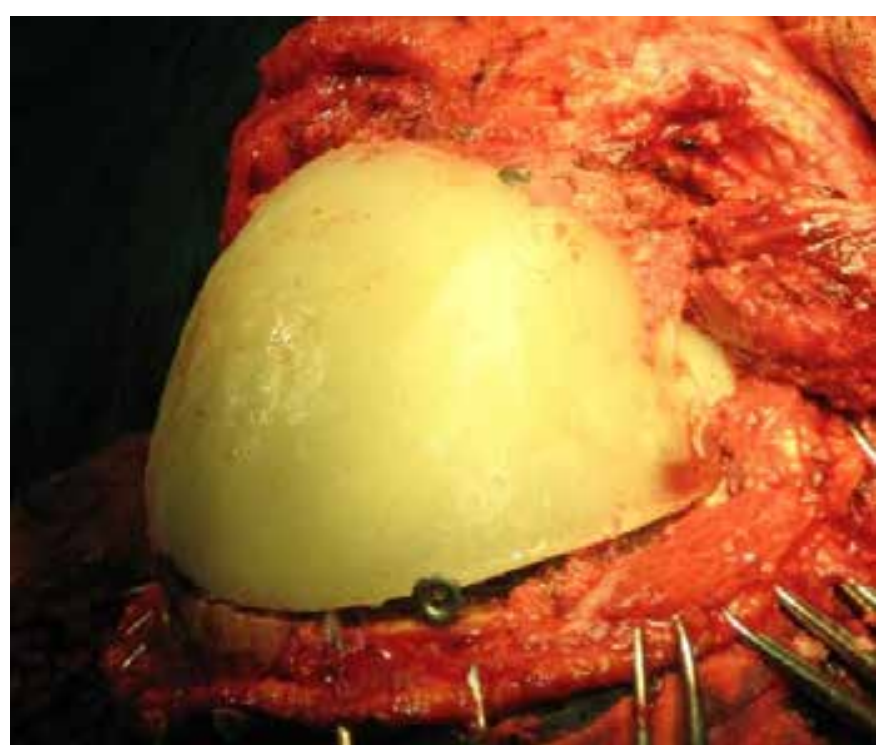

Figure 6: By using the planning template, a custom made silicon mould for the implant already existed and the defect closed with a cosmetically accurate implant.

All grafts were fitted into the defect without manipulation resulting in a $100 \%$ planning to implant ratio.

In the retrospective group, no septic complications were noted. One patient had an implant that displaced (too few fixation points resulting in swivelling) and in another an extradural hematoma was evacuated. This patient fell in the ward thus the hematoma was likely unrelated to the implant or procedure.

In the prospective group, patient demographic included 24 males and 6 females with a mean age of 31.9 years. Trauma was the most common cause of cranial vault defect (23 out of 30 patients) with 18 decompressive craniotomies. Five patients had emergency surgery for skull fractures that were contaminated and compromised bone was removed. Four patients had surgery to remove meningiomas. Of these, one extended intraosseously which was not appreciated preoperatively, two developed sepsis of the autologous bone 
immediately postoperatively and in one the bone was left off to control intracranial pressure. One patient presented with a subdural empyema with the bone left off and 2 developed wound sepsis after autologous bone was replaced for nontraumatic hematomas.

Cranioplasty was performed no sooner than 4 months after the initial surgery.

\section{Sepsis}

In total there were 5 infective complications resulting in an overall sepsis rate of $8.3 \%$. The pathogen involved in four of the cases was Staphylococcus Aureus with Group B haemolytic Streptococci being identified in one case.

All septic cases had superficial wound breakdown. No new osteitis was noted at the graft sites. There were no cases of deep seated infection. Of the 5 septic complications, 4 had initial primary septic pathologies (1 subdural empyema, 3 septic autologous bone flaps) and 1 was a meningioma. Two patients with sepsis had their implants removed due to leakage of cerebrospinal fluid from a dural breach.

There was no statistical significance between age, gender, time to surgery and sepsis rate, nor was there correlation between intraoperative blood loss or size and site of the craniotomy to sepsis rate.

\section{Cosmesis and Patient Satisfaction}

With regards to patient satisfaction, a three-point patient satisfaction scale was utilised. Twenty-three patients were satisfied, 2 were dissatisfied and 2 were neutral. Three patients were lost to follow-up. There was no correlation between age, gender and patient satisfaction. The two patients who were dissatisfied both developed septic complications.

\section{Cost}

The average cost of a moulded PMMA implant was at least 5 times cheaper than available similar products.

\section{Discussion}

The current public health service is overrun by the extensive trauma burden it faces on a day to day basis with trauma proving to be a massive challenge in the surgical environment. ${ }^{7}$ It poses a great challenge for cosmetic surgery when life-threatening conditions constantly invade surgical lists. Furthermore, should cosmetic type cases be done, elevated costs further add to them not being performed.

A cost-effective option exists in the form of PMMA which is widely used as bone cement in orthopaedic surgery. ${ }^{8}$ The challenges with attempting to create a cranioplasty implant by dealing directly with the PMMA paste is the different stages of hardening. Initially the paste is too soft and sticky to mould, and once the hardening effect is taking place, the heat element makes handling and shaping the implant very challenging with temperatures of up to $86^{\circ} \mathrm{C}$ reached. ${ }^{8}$ Numerous factors such as thickness of the graft, accurate interface with the defect edges and curvature play an extremely important role in ultimate cosmetic success.

The solution is to create a custom made implant based on a 3-dimensional reconstruction of the bony defect. This would meet the abovementioned criteria yet this could cost up to R200 000 per implant depending on the material with the cheapest options being around R75 000.

Significant cost-saving is achieved with the studied method of creating a custom silicon mould which is the negative of the desired implant that can be autoclaved in theatre. This gives the surgeon the ability to create a sterile implant (using PMMA) that perfectly matches the defect in all parameters. The mould has one or two so-called exhaust ports where excess PMMA can run off when the mould is compressed. This requires minor trimming of the graft prior to placement.

The study population was largely traumatic in nature and often when assaulted the patients' skin and underlying tissues are compromised which is a sepsis risk. Sepsis in cranioplasty is a well-known complication. Kwarcinski et al. in their meta-analysis of materials, manufacturing techniques and infection risk demonstrated an overall sepsis rate of $6.99 \%$ in pre-manufactured PMMA implants, $10.98 \%$ in hand-formed PMMA implants and $6.86 \%$ in templated PMMA implants. ${ }^{10}$ This was comparable with titanium mesh and plates which had $7.71 \%$ and $8.31 \%$ average reported infection rates. Infection risk was higher in cases which had previous infection or previous revision surgery. ${ }^{10}$

In our study, two cases of sepsis resulted from cerebrospinal fluid leakage causing superficial wound breakdown. One, on revision, was noted to be due to ingrowth of the temporal muscle into the dural patch of the previous surgery, and the other iatrogenic when the edges of the craniotomy site were freed from scar tissue and the dural defect not noted intraoperatively. One of the advantages of the silicon mould is the ability to re-use it if necessary as the mould is simply resterilised in theatre and a new PMMA implant created.

When dealing with previous craniotomy sites, care has to be taken when closing the wound with surgical clips as this can lead to subtle infolding of the wound edges pre-disposing it to sepsis. Closure of a previous site is recommended in these scenarios with Nylon. Fixation of the implant to the free bony edges can utilise any fixation devices but requires a minimum of 3 fixation points to prevent swivelling of the implant.

The use in delayed cranioplasty has proven to be very successful. A further use is in planning of cases where significant cranioplasty is anticipated. The preoperative 3D reconstructed skull is printed and the craniotomy planned on the model. A template is created to use as a guide for the craniotomy (sterilised in theatre) with a pre-determined mould ready to prepare the implant with. This creates a perfect cosmetic result and avoids further surgery (see Figures 4, 5 and 6).

\section{Conclusion}

Patient specific moulds using PMMA to create custom implants are safe, have excellent cosmetic results and are a very cost-effective option to manage cranial defects. Accurate planning strategies for large craniotomies, where bone will potentially be discarded, add to surgical effectiveness and cost saving to the patient. 


\section{REFERENCES}

1. Sorour M, Caton III WL, Couldwell WT. Technique for methyl methacrylate cranioplasty to optimize cosmetic outcome. Acta Neurochir (Wien). 2014;156(1):207- 9.

2. Sundseth J, Sundseth A, Berg-Johnsen J, Sorteberg W, Lindegaard K-F. Cranioplasty with autologous cryopreserved bone after decompressive craniectomy. Complications and risk factors for developing surgical site infection. Acta Neurochir (Wien). 2014;156:805-11.

3. Breusch SJ, Malchau H. The Well-cemented Total Hip Arthroplasty: Theory and Practice. Heidelberg, NY: SpringereBerlin, 2005; p. 52-66.Becker LC, Bergfeld WF, Belsito DV, et al. Final report of the cosmetic ingredient review expert panel safety assessment of Polymethyl Methacrylate (PMMA),Methyl Methacrylate Crosspolymer, and Methyl Methacrylate/Glycol Dimethacrylate Crosspolymer. Int J Toxicol. 2011;30(3 Suppl):54S-65S.

4. Kim BJ,; Hong KS, Park KJ, Park DH, Chung YG, Kang SH. Customized cranioplasty implants using three-dimensional printers and polymethyl-methacrylate casting. J. Korean Neurosurg Soc. 2012;52:541-546.

5. Marbacher S, Andereggen L, Erhardt S, Fathi AR, Fandino J, Raabe A, Beck J. Intraoperative template-molded bone flap reconstruction for patient-specific cranioplasty. Neurosurg Rev. 2012;35:527-35.

6. Walker I, Vlok AJ, Kamat A. A double-edged sword: the effect of technological advancements in the management of neurotrauma patients. Br J Neurosurg. 2017;31(1):89-93.

7. Gooch MR, Gin GE, Kenning TJ, German JW. Complications of cranioplasty following decompressive craniectomy: Analysis of 62 cases. Neurosurg Focus. 2009;26:E9.

8. Kim BJ, Hong KS, Park KJ, Park DH, Chung YG, Kang SH. Customized cranioplasty implants using three-dimensional printers and polymethyl-methacrylate casting. J. Korean Neurosurg Soc. 2012;52:541-6.

9. Available from: www.eppleyplasticsurgery.com/skull-reshaping (Accessed 10 July 2017).

10. Vaishya R, Chauhan M, Vaish A. Bone cement. J Clin Orthop Trauma. 2013;4(4): 157-63.

11. Kwarcinski J, Boughton P, Ruys A, Doolan A, Van Gelder, J. Cranioplasty and Craniofacial Reconstruction: A Review of Implant Material, Manufacturing Method and Infection Risk. Appl Sci. 2017;7:276.

12. Fathi AR, Marbacher S, Lukes A. Cost-effective patientspecific intraoperative molded cranioplasty. J Craniofac Surg. 2008;19:777-81. 